\title{
Depauperate genetic variability detected in the American and European bison using genomic techniques
} Cino Pertoldi ${ }^{* 1,2}$, Macgorzata Tokarska ${ }^{1}$, Jan M Wójcik ${ }^{1}$, Ditte Demontis ${ }^{2}$, Volker Loeschcke2, Vivi R Gregersen ${ }^{3}$, David Coltman ${ }^{4}$, Gregory A Wilson ${ }^{4,5}$, Ettore Randi ${ }^{6}$, Michael M Hansen ${ }^{2}$ and Christian Bendixen ${ }^{3}$

Address: ${ }^{1}$ Mammal Research Institute, Polish Academy of Sciences, Waszkiewicza 1c, 17-230 Biacowie $i$ a, Poland, ${ }^{2}$ Department of Biological Sciences, Ecology and Genetics, Aarhus University, Ny Munkegade, Building 1540, 8000 Århus C, Denmark, ${ }^{3}$ Department of Genetics and Biotechnology, Faculty of Agricultural Sciences, Aarhus University, PO Box 50, DK-8830 Tjele, Denmark, ${ }^{4}$ Department of Biological Science, University of Alberta, Ctr Biol Sci, Edmonton, AB T6G 2E9, Canada, ${ }^{5}$ Canadian Wildlife Service, 200, 4999 - 98 Ave, Edmonton, AB T6B 2X3, Canada and ' $I$ stituto Superiore per la Protezione e la Ricerca Ambientale, via Cá Fornacetta 9, I-40064, Ozzano Emilia (BO), Italy

Email: Cino Pertoldi* - biocp@nf.au.dk; Macgorzata Tokarska - tokarska@bison.zbs.bialowieza.pl; Jan M Wójcik - jwojcik@zbs.bialowieza.pl; Ditte Demontis - biodha@nf.au.dk; Volker Loeschcke - biovl@nf.au.dk; Vivi R Gregersen - Vivi.Gregersen@agrsci.dk;

David Coltman - david.coltman@ualberta.ca; Gregory A Wilson - Greg.Wilson@EC.gc.ca; Ettore Randi - ettore.randi@infs.it; Michael M Hansen - mmh@biology.au.dk; Christian Bendixen - Christian.Bendixen@agrsci.dk

* Corresponding author

Published: 8 December 2009

Biology Direct 2009, 4:48 doi:10.1186/1745-6150-4-48

This article is available from: http://www.biology-direct.com/content/4/1/48

(C) 2009 Pertoldi et al; licensee BioMed Central Ltd.

This is an Open Access article distributed under the terms of the Creative Commons Attribution License (http://creativecommons.org/licenses/by/2.0), which permits unrestricted use, distribution, and reproduction in any medium, provided the original work is properly cited.

\begin{abstract}
: A total of 929 polymorphic SNPs in EB (out of 54, 000 SNPs screened using a BovineSNP50 Illumina Genotyping BeadChip), and I, 524 and I, 403 polymorphic SNPs in WB and PB, respectively, were analysed. $\mathrm{EB}, \mathrm{WB}$ and $\mathrm{PB}$ have all undergone recent drastic reductions in population size. Accordingly, they exhibited extremely depauperate genomes, deviations from genetic equilibrium and a genome organization consisting of a mosaic of haplotype blocks: regions with low haplotype diversity and high levels of linkage disequilibrium. No evidence for positive or stabilizing selection was found in EB, WB and PB, likely reflecting drift overwhelming selection. We suggest that utilization of genome-wide screening technologies, followed by utilization of less expensive techniques (e.g. VeraCode and Fluidigm EPI), holds large potential for genetic monitoring of populations. Additionally, these techniques will allow radical improvements of breeding practices in captive or managed populations, otherwise hampered by the limited availability of polymorphic markers. This result in improved possibilities for I) estimating genetic relationships among individuals and 2) designing breeding strategies which attempt to preserve or reduce polymorphism in ecologically relevant genes and/or entire blocks.
\end{abstract}

Reviewers: This article was reviewed by: Fyodor Kondrashov and Shamil Sunyaev

\section{Findings}

Fifty-four EB (Bison bonasus bonasus), $30 \mathrm{WB}$ (Bison bison athabascae) and $26 \mathrm{~PB}$ (Bison bison bison) were used for genome screening. The PB samples derive from Elk Island
National Park while WB samples come from Wood Buffalo National Park (Canada). The relationships (mother, father and offspring) of three EB families were used in the SNP screening to verify Mendelian segregation. Genome- 
wide screening encompassed 54, 000 single nucleotide polymorphisms (SNPs) across the entire bovine genome. More than half of the SNPs were discovered using the sequencing system Genome Analyzer (Illumina ${ }^{\circledR}$ ) [1]. The additional SNP content was derived from publicly available sources such as Btau (ftp://ftp.hgsc.bcm.tmc.edu/pub/ data/Btaurus/fasta), the bovine reference genome, and the Bovine HapMap Consortium data set (). The BeadChip has an MAF of 0.25 across all loci and has been validated in both dairy and beef cattle. SNPs were genotyped on the BovineSNP50 BeadChip according to the Infinium II Multi-Sample assay protocol provided by Illumina (Manual Experienced User Card, 11208000 Rev. A., Illumina Inc.).

Despite the fact that the Bos and Bison lineages split about 1 million years ago [2], a total of 2, 209 polymorphic SNPs were found when EB, PB and WB were pooled. The average call rate for the bison samples was relatively high (96.60\%) compared to the call rate found for the cattle samples (99.5\%) (Illumina, Inc. Pub No. 370-2007-029) [1]. The small difference in call rate $(2.9 \%)$ confirms the reliability of our comparisons and also reflects differences in the genomic DNA between the two groups such as the possibility of different alleles or deletions in SNPs.

The $\mathrm{P} \%$ for each bison species was calculated relative to the total number of loci that were polymorphic when EB, $\mathrm{WB}$ and $\mathrm{PB}$ samples were pooled. The pairwise $\mathrm{F}_{\mathrm{ST}}$ used as a measure of differentiation between $\mathrm{EB}, \mathrm{PB}$ and $\mathrm{WB}$ was estimated using FSTAT version 2.9.3 [3] (). Genetic differentiation between $\mathrm{PB}$ and $\mathrm{WB}$ was significant $\left(\mathrm{F}_{\mathrm{ST}}=\right.$ $0.114)$, albeit much lower compared to EB-PB $\left(\mathrm{F}_{\mathrm{ST}}=\right.$ $0.466)$ or EB-WB $\left(\mathrm{F}_{S T}=0.474\right)$. Genetic differentiation estimated between $\mathrm{EB}, \mathrm{WB}$ and $\mathrm{PB}$ might provide a relative estimate of the time elapsed since divergence in isolation.

The number of mapped, polymorphic SNPs in EB was 929 $(\mathrm{P} \%=42 \%)$. The distribution of these markers along the autosomal chromosomes (on the corresponding known position on the 29 autosomal bovine chromosomes) is shown in Figure 1. More polymorphic SNPs were found in $\mathrm{WB}(\mathrm{P} \%=69 \%)$ and $\mathrm{PB}(\mathrm{P} \%=63 \%)(1,524$ and 1,403 SNPs, respectively, see Figures 2 and 3 ).

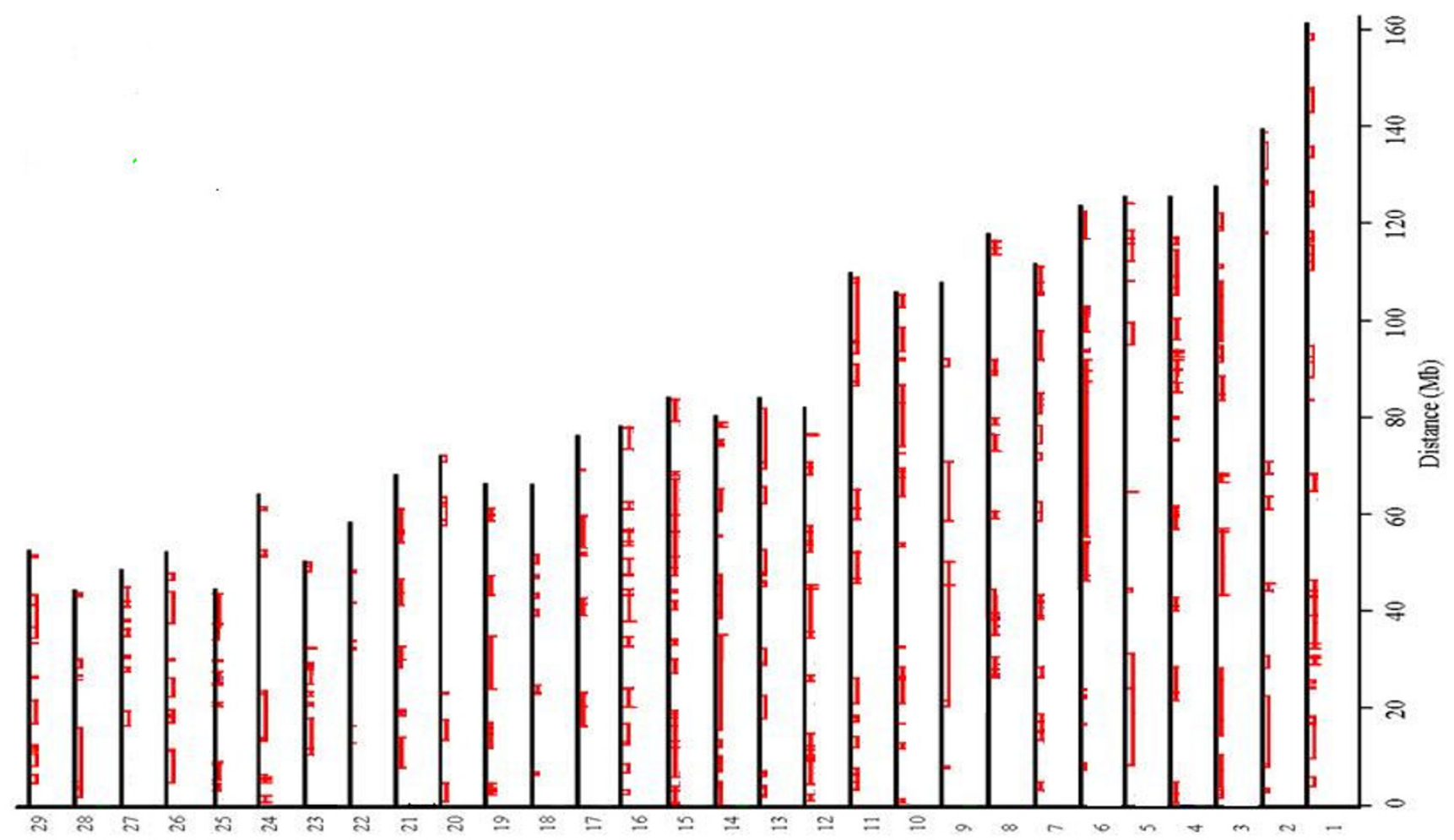

Figure I

The distribution of the 929 polymorphic SNPs in EB and haplotype block partitioning. Hatch marks of all SNPs in a certain block are connected by a line. Each hatch represents a SNP. 


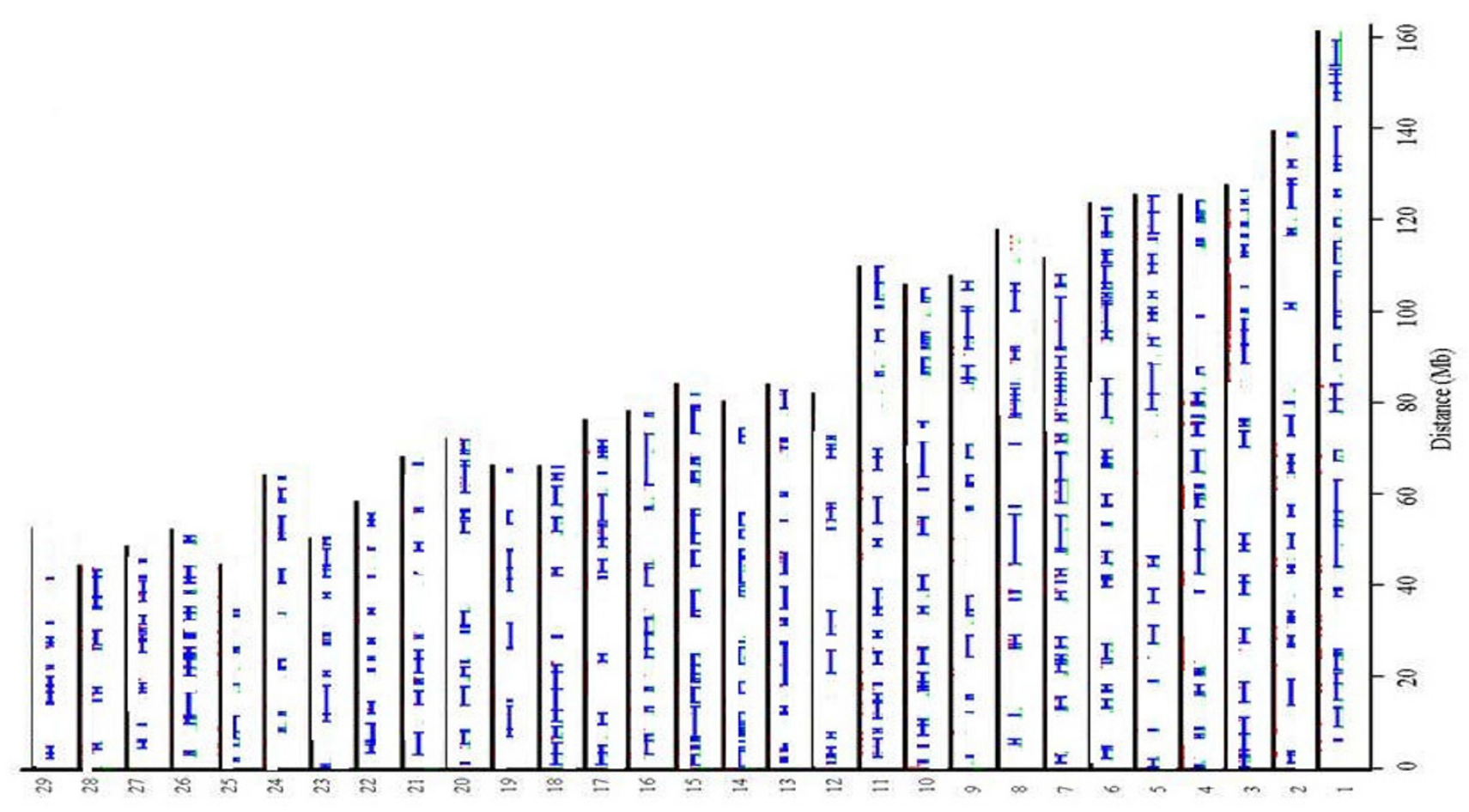

\section{Figure 2}

The distribution of the I, 524 polymorphic SNPs in WB and haplotype block partitioning. Hatch marks of all SNPs in a certain block are connected by a line. Each hatch represents a SNP.

After considering the distribution of the distance between the polymorphic loci the haplotypes were calculated according to the four gamete rule implemented in the Haploview 4.1 software [4].

Inspection of the polymorphic SNPs (see Figure 1, 2 and 3 ) reveals long chromosomal regions fixed for one allele. Our findings have some similarities with a study of the cattle genome [5] which consists mainly of a mosaic of haplotype blocks, i.e. regions with low haplotype diversity and high levels of linkage disequilibrium [5]. These haplotype blocks may reflect several factors, including genetic hitchhiking, variable mutation rates and recombination, gene flow, drift, inbreeding and lack of genetic equilibrium [6]. Therefore, the processes underlying these haplotype blocks should be interpreted with caution. However, it is likely that many of the shared blocks and, in turn, highly polymorphic regions are ancestral. Hence, more detailed screening could answer important evolutionary questions.

Very few segregating SNPs were shared between EB and PB $(1.44 \%)$ and $\mathrm{EB}$ and WB $(1.89 \%)$ when compared to the overlap between PB and WB (41.91\%). The number of shared polymorphisms is inversely related to the estimated $\mathrm{F}_{\mathrm{ST}}$ values, confirming that they are related to the time elapsed since divergence in isolation of $\mathrm{EB}, \mathrm{PB}$ and WB.

Simulations made with the software BOTTLENECK 1.2.02 [7] assuming an infinite alleles mutation model, showed decreased abundance of low frequency alleles $(<0.1)$ relative to intermediate allele frequency classes $(0.1-0.2,0.2$ $0.3,0.3-0.4)$ in $\mathrm{WB}$ and $\mathrm{PB}$, whereas for EB the distribution was L-shaped. This mode-shift in the distribution of allele frequencies in $\mathrm{WB}$ and $\mathrm{PB}$ signifies population bottlenecks. The tests indicate therefore that $\mathrm{WB}$ and $\mathrm{PB}$ are not in genetic equilibrium (as expected after recent demographic declines). However, the EB population is in genetic equilibrium, despite the documented recent, strong bottleneck. This unexpected result could be due to the fact that the bottleneck in EB may have reduced $\mathrm{N}_{e^{\prime}}$ which is negatively correlated to the speed at which the population re-establishes mutation-drift equilibrium and show therefore an L-shaped distribution. Given the distortion of the L-shaped distribution is transient, and likely to be detectable until genetic drift begin to re-establish mutation-drift equilibrium [7] we hypothesize that the 


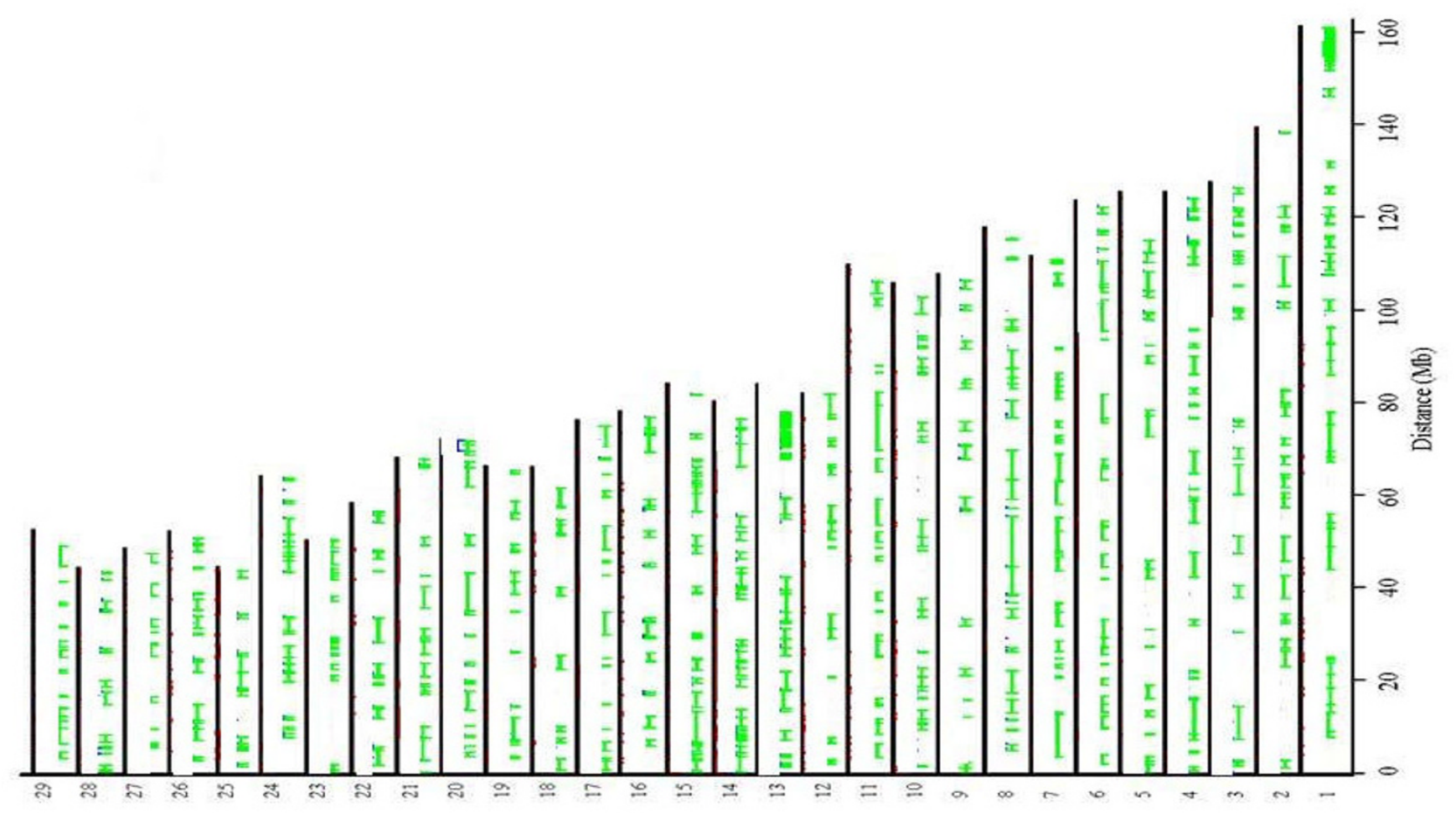

Figure 3

The distribution of the I, 403 polymorphic SNPs in PB and haplotype block partitioning. Hatch marks of all SNPs in a certain block are connected by a line. Each hatch represents a SNP.

extremely small $\mathrm{N}_{\mathrm{e}}$ produced by the drastic bottleneck in $\mathrm{EB}$, has allowed a rapid return of the population to genetic equilibrium.

As a consequence of the finding that $\mathrm{WB}$ and $\mathrm{PB}$ are not at genetic equilibrium we must expect that the relation between $\mathrm{F}_{\mathrm{ST}}$ and divergence time between $\mathrm{EB}, \mathrm{WB}$ and $\mathrm{PB}$ can be strongly biased. Additionally, the presence of blocks in the genome can probably partially be explained by this lacks of genetic equilibrium. Even if the EB population is now at equilibrium, we cannot exclude that previous bottlenecks have contributed to the observed pattern of a mosaic of haplotype blocks.

The presence of a mosaic blocks pattern and evidences for a mode-shift in the distribution of allele frequencies found in WB and PB agrees with theoretical expectations for populations which have undergone a severe bottleneck [8]. The finding that $\mathrm{P} \%$ in $\mathrm{EB}$ is relatively smaller than $\mathrm{P} \% \mathrm{~PB}$ and $\mathrm{WB}$, is not surprising as the European bison underwent the most extreme bottleneck in population size of the three groups at the beginning of the last century; EB stems from only 7 animals [8]. Consequently, due to the founder effect, the current free-living Biacowie $;$ a population of the European bison (about 400 individuals in 2007) is expected to have lower genetic diversity than the historic population. $\mathrm{PB}$ and $\mathrm{WB}$ have also experienced bottlenecks $[9,10]$, of about 150 individuals in PB [11] and 250 in WB [12]. During the last 100 years the population size has increased and census population sizes are now estimated to be large both for $\mathrm{PB}$ and WB, although most of the bison in North America occur in commercial herds [10]. Despite rapid population growth in the last century, $\mathrm{N}_{e}$ has therefore increased slowly in bison. This is because the long term $\mathrm{N}_{e}$ is a function of the harmonic mean which is strongly influenced by the minimum population size reached $[13,14]$.

In order to detect regions of bison chromosomes which show signs of positive selection we checked for the existence of "outlier SNPs" by creating distributions of $\mathrm{H}_{\mathrm{E}}$ for every locus in EB, PB and WB. The SNPs were considered outliers if their $\mathrm{H}_{\mathrm{E}}$ was $>$ mean $\mathrm{H}_{\mathrm{E}}+3 \mathrm{SD}$ or $<$ mean $\mathrm{H}_{\mathrm{E}}$ 3SD. These tests were performed pooling all loci and testing every single chromosome singularly. Additionally, we tested (with a $t$-test) for $\mathrm{EB}, \mathrm{WB}$ and $\mathrm{PB}$ if the $\mathrm{H}_{\mathrm{E}}$ estimated from the polymorphic SNPs shared among EB, WB and PB were significantly different from the $\mathrm{H}_{\mathrm{E}}$ estimated from 
the unshared polymorphic SNPs. These tests were conducted because we suspected that the reasons for this shared polymorphism could be due to stabilizing selection. However, no significant differences were found. The lack of evidence for positive selection in $\mathrm{EB}, \mathrm{WB}$ and $\mathrm{PB}$ is not surprising as the selection intensity has to be larger than $\frac{1}{2 N_{e}}$ for selection to be efficient $[15,16]$. Given that $\mathrm{N}_{\mathrm{e}}$ is low for bison in general, all SNPs, even if not selectively neutral, behave neutrally until $\mathrm{N}_{\mathrm{e}}$ reaches a certain threshold.

By screening a large number of SNPs we considerably reduce the sampling error of the estimated overall genomic variability compared to traditional investigations that use a much more limited number of markers (SNPs or microsatellites). The SNPs can also be used in more limited numbers for genetic monitoring of populations. For many years, microsatellites have been the markers of choice for this purpose, using non-invasive techniques like extraction of DNA from scat or other organic material, and also using historical samples in order to add a temporal dimension to the survey. However, non-invasive DNA samples are often of poor quality and prone to genotyping errors due to DNA degradation, making long genetic markers like microsatellites difficult to use. Conversely, SNPs have the advantage that they are able to amplify short DNA sequences, decreasing therefore artifacts occurring due to degraded DNA. Additionally, the information from the BeadChip might allow genome-wide based breeding schemes designed to preserve rare alleles and minimize inbreeding (by estimating the true relationships between individuals). Traditional methods for making breeding decisions to reduce the level of inbreeding (by increasing $\mathrm{N}_{e}$ ) utilize only pedigree information, which describes the expected relationship among individuals. With the same pedigree, however, individuals still vary in the realized genetic relationship among them [17]. Therefore, information obtained from genetic markers can be useful in this respect as they will provide the realized genetic relationships.

The fact that the genome of $\mathrm{EB}, \mathrm{WB}$ and $\mathrm{PB}$ is organised in blocks allows for designing breeding strategies which can use genotype data instead of pedigree data and which have the scope to increase or reduce the polymorphism of the blocks. The fact that there are several polymorphic SNPs embedded in the blocks (see fig. 1, 2 and 3), allow us to reduce considerably the number of SNPs which need to be screened in order to apply a breeding strategy. In fact, our results suggest that one single SNP can detect the level of polymorphism of an entire block. Hence, choosing a subset of the polymorphic SNPs (48 or 96 SNPs) we can apply relatively cheap technologies such as VeraCode or Fluidigm EP1 system (). With the information obtained from the BeadChip it will be possible to create a SNP panel on the polymorphisms described here and use them in a MAS strategy [18].

Usage of a SNP panel as proposed here would allow not only reliable parentage and identity analysis but would also provide a standardized panel for exchange of genotype data between laboratories. As the range of off-theshelf SNP genotyping systems grows, they may find increased use for a number of endangered species (and rare domestic breeds). Further research is needed to determine if existing SNP genotyping systems can be applied across whole taxonomic orders or families, or if they are only transferable at the species or genus level, and to what extent screening success depends on taxonomic distance from the source taxon or population history of the study taxon. A problem often associated with SNPs application in population studies is ascertainment bias $[19,20]$. It is generated by heterogeneity in the SNP discovery process, variable sample size and composition, and may cause underestimation or overestimation of globally distributed SNP frequencies [20]. Since the chip markers used in our survey were selected for cattle, the possible ascertainment bias could appear when comparing the SNP based genetic variability of cattle and bison. Thus, a bison - cattle comparisons should be interpreted with caution. However the random selection of markers should not constitute a problem when the European bison is the only subject of an inquiry thus it was not considered in our study.

\section{List of abbreviations}

EB: European bison; $\mathrm{H}_{\mathrm{E}}$ : expected heterozygosity; MAF: Minor allele frequency; MAS: Marker assisted selection; $\mathrm{N}_{e}$ : Effective population size; P\%: Percent of polymorphic loci; PB: Plain bison; S: Selection gradient; WB: Wood bison

\section{Competing interests}

The authors declare that they have no competing interests.

\section{Authors' contributions}

All authors have conceived the study and drafted the manuscript. All authors have read and approved the final manuscript.

\section{Reviewers' comments Reviewers' report I}

Fyodor Kondrashov (Centre for Genomic Regulation Barcelona Spain).

\section{Reviewers' comments}

This reviewer provided no comments for publication 


\section{Reviewers' report 2}

Shamil Sunyaev (Harvard Medical School, Boston MA, United States).

\section{Reviewers' comments}

This is an interesting study of genetic variation in bison. The authors characterized variation in the European bison and American wood and plains bisons using the BovineSNP50 Illumina Genotyping BeadChip. I have three comments listed below: 1)The manuscript seems to suggest that the observed small number of SNPs from the BovineSNP50 chip segregating in the bison population reflects a population bottleneck. However, the authors measured the level of shared polymorphism between bison and cow rather than the overall levels of variation in bison. The level of shared polymorphism has a more complex relationship with $\mathrm{N}_{e}$ and also depends on other parameters (for the theoretical treatment see Clark AG. Neutral behaviour of shared polymorphism. Proc Natl Acad Sci U S A. 1997 94:7730-4.) Thus, a more sophisticated discussion of the observations would be appropriate instead of simply stating the low level of variation and, subsequently, $\mathrm{N}_{\mathrm{e}}$.

\section{Author's response}

We agree with the Reviewer that it would have been interesting to estimate the $N_{e}$ following the method presented in Clark (1997). This method is based on the fact that the loss of a shared polymorphism of a neutral allele having an initial allele frequency of 1/2 occurs sooner than loss of polymorphism in a single species and has an expected time of $1.7 \mathrm{~N}_{e}$ generations, compared to $2.77 \mathrm{~N}_{e^{\prime}}$ which is the mean time necessary for one neutral allele to undergo fixation within a single population. Therefore, given the fact that in our study we have found several shared polymorphisms, we would have been able to estimate the historical $N_{e}$ of EB, WB and PB. However, the method presented by Clark requires that the loci utilised for the estimation of $N_{e}$ are unlinked. Hence, we performed tests for linkage disequilibrium and ran the program Haploview 4.1 which showed that the genome of the bison is mainly a mosaic of haplotype blocks where strong linkage disequilibrium between SNPs is observed (see please the last paragraph on page 5 and please see fig. 1, 2 and 3 where the blocks are shown graphically). Therefore, we have not been able to use Clark's method, but we decided to utilise the shared polymorphism in order to test if $H_{E}$ within a subspecies (pooling all the SNPs with shared polymorphism) was significantly different from $\mathrm{H}_{E}$ within a subspecies (pooling all the SNPs which are not shared among EB, WB and $P B)$. The differences of mean $H_{E}$ was not significant. Therefore, we did not obtain evidence for the fact that the shared polymorphism could be due to stabilizing selection acting on these SNPS (please see the first paragraph on page 8).
2) The manuscript would benefit from the analysis of allele frequency distribution (excess of high frequency variants may be suggestive of a bottleneck) and $F_{S T}$ between the analyzed populations.

\section{Author's response}

Population bottlenecks cause a characteristic mode-shift distortion in the distribution of allele frequencies at selectively neutral loci. In a stable population the distribution is expected to be L-shaped. Bottlenecks cause alleles at low frequency $(<0.1)$ to become less abundant than alleles in more intermediate allele frequency classes (e.g., 0.1-0.2). We performed simulations with the software BOTTLENECK 1.2.02, assuming an infinite allele mutation model searching for mode-shift distortion in the distribution of allele frequencies. We found evidence for lack of genetic equilibrium in WB and PB (see please the third paragraph on page 5). Furthermore, the pairwise $F_{S T}$ values as a measure of differentiation between $E B, P B$ and $W B$ have been added (see please the first paragraph on page 5).

3) Is it possible to quantify the improvement of breeding strategies using genotyping instead of pedigree data?

\section{Author's response}

An exact quantification of the improvement of breeding strategy can only be made using empirical data comparing the improvement of a certain trait in mating individuals chosen on the basis of their pedigree or on the basis of their genetic similarity at the molecular level. Traditional methods for making breeding decisions to reduce the level of inbreeding (by increasing $N_{e}$ ) utilize only pedigree information, which describes the expected relationship among individuals. With the same pedigree, however, individuals still vary in the realized genetic relationship between them. Therefore, information obtained from genetic markers can be useful in this respect as they will provide the realized genetic relationships. Additionally, information from genome-wide screening also enables detection of genes associated with hereditary genetic diseases and inbreeding depression (these issues have been discusses in the last paragraph on page 8).

\section{Acknowledgements}

This study has been partly supported by a Marie Curie Transfer of Knowledge Fellowship BIORESC of European Community's Sixth Framework Programme (contract number MTKD-CT-2005-029957), by the Frankfurt Zoological Society - Help for Threatened Wildlife and by the LIFE financial instrument of the European Community (project "Bison Land - European bison conservation in Biacowie ¿a Forest", LIFE06 NAT/PL/000I05 BISONLAND). Furthermore we wish to thank the ConGen program (funded by the European Science Foundation) and the Danish Natural Science Research Council for financial support to CP (grant number: \#2 I-01-0526, \#21-03-0125 and 95095995) and Torsten N Kristensen for helpful comments on the ms. 


\section{References}

I. Tokarska M, Marshall T, Kowalczyk R, Wojcik JM, Pertoldi C, Kristensen TN, Loeschcke V, Gregersen VR, Bendixen C: Effectiveness of microsatellite and SNP markers for parentage and identity analysis in species with low genetic diversity: the case of European bison. Heredity 103:326-332.

2. Loftus RT, MacHugh DE, Bradley DG, Sharp PM, Cunningham EP: Evidence for two independent domestications of cattle. Proc Natl Acad Sci USA 1994, 9 1:2757-2761.

3. Goudet J: FSTAT Version I.2: a computer program to calculate F-statistics. J Heredity 1995, 86:485-486.

4. Barrett JC, Fry B, Maller J, Daly MJ: Haploview: analysis and visualization of LD and haplotype maps. Bioinformatics 2005, 2I:263-265

5. Khatkar MS, Zenger KR, Hobbs M, Hawken R J, Cavanagh J AL, Barris W, McClintock A E, McClintock S, Thomson P C, Tier B, Nicholas F W, Raadsma $\mathrm{H}$ W: A primary assembly of a bovine haplotype block map based on a I $5 \mathrm{k}$ SNP panel genotyped in HolsteinFriesian cattle. Genetics 2007, I 74:79-85.

6. Tenesa A, Navarro P, Hayes BJ, Duffy DL, Clarke GM, Goddard ME, Visscher PM: Recent human effective population size estimated from linkage disequilibrium. Genome Res 2007, 17:520-526

7. Cornuet JM, Luikart G: Description and power analysis for two tests for detecting recent population bottlenecks from allele frequency data. Genetics 1996, I44:200I-2014.

8. Nei M, Maruyama T, Chakraborty R: The bottleneck effect and genetic variability in populations. Evolution $1975,29: 1-10$.

9. Pucek Z, Belousova IP, Krasieska M, Krasieski Z, Olech W: European Bison Status Survey and Conservation Action Plan. IUCN/SSC Bison Specialist Group. Gland and Cambridge: IUCN; 2004.

10. Halbert ND, Raudsepp T, Chowdhary BP, Derr JN: Conservation genetic analysis of the Texas state bison herd. J Mamm 2004, 85:924-931.

II. Freese $\mathrm{CH}$, Aune KE, Boyd DP, Derr NJ, Forrest SC, Gates CC, Gogan PJP, Grassel SM, Halbert ND, Kunkel K, Redford KH: Second chance for the plains bison. Biol Conserv 2007, I36:175-184.

12. Wilson GA, Strobeck C: Genetic variation within and relatedness among wood and plains bison populations. Genome 1999:483-496

13. Pertoldi C, Bach LA, Loeschcke V: On the brink between extinction and persistence. Biology Direct 2008, 3:47.

14. Pertoldi C, Bach LA, Barker JSF, Lundberg P, Loeschcke V: The consequences of the variance-mean rescaling effect on effective population size. OIKOS 2007, 5:769-774.

15. Pertoldi C, Bijlsma R, Loeschcke V: Conservation genetics in a globally changing environment: present problems, paradoxes and future challenges. Biodivers Conserv 2007 16:4|47-4|63.

16. Pertoldi C, Bach L: Evolutionary aspects of climate induced changes and the need for multidisciplinarity. J Therm Biol 2007, 32: $118-124$.

17. Nielsen RK, Pertoldi C, Loeschcke V: Genetic evaluation of the captive breeding program of the Asiatic wild ass, onager (Equus hemionus onager). J Zool 2007, 272:349-357.

18. Wang J, Hill WG: Marker assisted selection to increase effective population size by reducing Mendelian segregation variance. Genetics 2000, I 54:475-489.

19. Nielsen R, Signorovitch J: Correcting for ascertainment biases when analyzing SNP data: applications to the estimation of linkage disequilibrium. Theoretical Population Biology 2003, 63:245-255.

20. Clark AG, Hubisz MJ, Bustamante CD, Williamson $\mathrm{SH}$, Nielsen R: Ascertainment bias in studies of human genome-wide polymorphism. Genome Research 2005, I5:|496-I502.

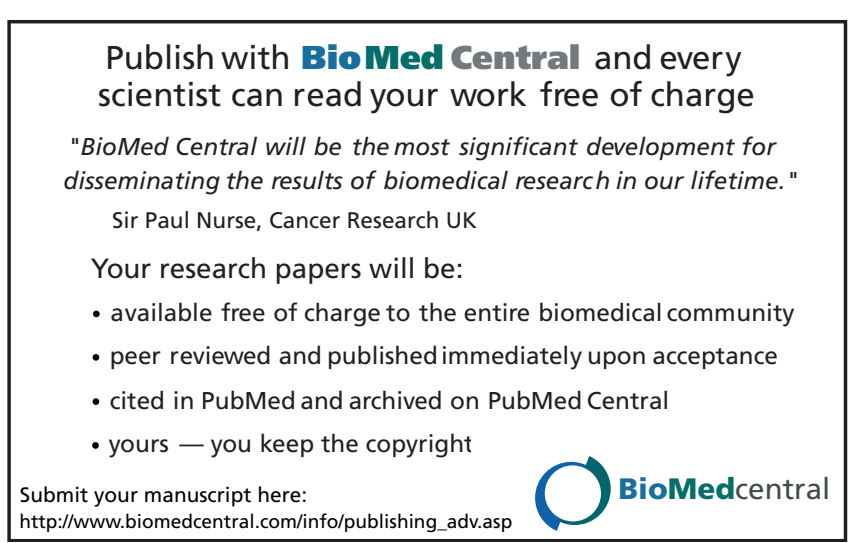

